\title{
CÁC NHÂN TỐ ẢNH HƯỞNG TỚI HOẠT ĐỘNG NGHIÊN CÚU VÀ PHÁT TRIỂN - ĐÁNH GIÁ TƯ GÓC ĐỘ NHÀ QUẢN LÝ Ở CÁC TRƯỜNG ĐẠI HỌC KHỐI KỸ THUẠT, CÔNG NGHỆ
}

\author{
FACTORS AFFECTING RESEARCH AND TECHNOLOGY \\ DEVELOPMENT ACTIVITIES - A STUDY FROM \\ OPINIONS OF ADMINISTRATORS IN TECHNOLOGY \\ AND TECHNICAL UNIVERSITIES
}

\author{
Nguyễn Đăng Tuệ ${ }^{1}$ \\ Ngày nhận bài: 16/6/2019 Ngày chấp nhận đăng: 01/8/2019 Ngày đăng: 05/12/2019
}

\section{Tóm tắt}

Hoạt động phát triển khoa học và công nghệ đóng vai trò quan trọng đối với các trường đại học trong việc nâng cao chất lượng đào tạo và xây dựng danh tiếng của trường. Nghiên cứu này tìm hiểu các nhân tố ảnh hưởng đến kết quả của hoạt động phát triển khoa học và công nghệ thông qua đánh giá của các nhà quản lý. Sử dụng bộ số liệu điều tra của Hiệp hội cá trường đại học và cao đẳng Việt Nam, tác giả áp dụng mô hình cấu trúc tuyến tính (SEM) để kiểm định các giả thuyết liên quan đến sự tác động của các nhóm nhân tố quản lý, trao đổi thông tin, tài chính và nhân lực tới kết quả của hoạt động phát triển khoa học và công nghệ. Kết quả cho thấy các nhóm nhân tố quản lý, nhân lực có ảnh hưởng tới kết quả hoạt động phát triển khoa học và công nghệ và nhóm nhân tố tài chính là cản trở đối với hoạt động này. Dựa trên kết quả thu được, tác giả đưa ra một số hàm ý chính sách.

Tù khóa: Khoa học công nghệ, nhân tố, quản lý, trường đại học, Việt Nam.

\begin{abstract}
Research and technology development plays a vital role in improving universities' quality and enhancing their reputation. This research explores factors affecting research and technology development activities by analyzing opinions of administrators in technology and technical universities. Using survey data of the Association of Vietnam Universities and Colleges, the authors apply structural equation modeling (SEM) to test the hypotheses about whether management, communication, financial and human resource affect the results of research and technology development activities. The results indicate management and human resource factors can positively affect research and technology development activities while financial factors can significantly hinder these activities. Based on the findings, the authors suggest some policy implications Keywords: Science, technology, factors, management, university, Vietnam.
\end{abstract}

\footnotetext{
${ }^{1}$ Viện Kinh tế và Quản lý, Trường đại học Bách khoa Hà Nội, Email: nguyendangtue@gmail.com
} 


\section{Bối cảnh nghiên cứu}

Luật Khoa học và công nghệ Việt Nam năm 2013 đã xác định hoạt động nghiên cứu và phát triển (NC\&PT) bao gồm: (1) Nghiên cứu khoa học là hoạt động khám phá, phát hiện, tìm hiểu bản chất, quy luật của sự vật, hiện tượng tự nhiên, xã hội và tư duy; sáng tạo giải pháp nhằm ứng dụng vào thực tiễn; (2) Nghiên cứu cơ bản là hoạt động nghiên cứu nhằm khám phá bản chất, quy luật của sự vật, hiện tượng tự nhiên, xã hội và tư duy; (3) Nghiên cứu ứng dụng là hoạt động nghiên cứu vận dụng kết quả nghiên cứu khoa học nhằm tạo ra công nghệ mới, đổi mới công nghệ phục vụ lợi ích của con người và xã hội; (4) Phát triển công nghệ là hoạt động sử dụng kết quả nghiên cứu cơ bản, nghiên cứu ứng dụng, thông qua việc triển khai thực nghiệm và sản xuất thử nghiệm để hoàn thiện công nghệ hiện có, tạo ra công nghệ mới; (5) Triển khai thực nghiệm là hoạt động ứng dụng kết quả nghiên cứu khoa học và phát triển công nghệ để tạo ra sản phẩm công nghệ mới ở dạng mẫu; (6) Sản xuất thử nghiệm là hoạt động ứng dụng kết quả triển khai thực nghiệm để sản xuất thử nhằm hoàn thiện công nghệ mới, sản phẩm mới trước khi đưa vào sản xuất và đời sống. Các hoạt động NC\&PT đã được thực hiện tích cực tại các trường đại học Việt Nam, đặc biệt là tại các trường đại học khối kỹ thuật, công nghệ. Văn kiện Đại hội đại biểu toàn quốc lần thứ XII của Đảng Cộng sản Việt Nam (Dự thảo Báo cáo đánh giá kết quả thực hiện nhiệm vụ phát triển KT-XH 5 năm 2011-2015 và phương hướng, nhiệm vụ phát triển KTXH 5 năm 2016-2020 trình Đại hội đại biểu toàn quốc lần thứ XII của Đảng Cộng sản Việt Nam) đã đưa ra các nhiệm vụ và giải pháp về phát triển KH\&CN giai đoạn 2016-2020, trong đó bao gồm nội dung liên quan đến tăng cường hoạt động nghiên cứu khoa học trong các trường đại học như đổi mới cơ chế quản lý, nhất là cơ chế tự chủ về tài chính, tổ chức và hoạt động của tổ chức khoa học, công nghệ công lập (trong đó có các trường đại học); có cơ chế đặc thù để phát triển các cơ sở nghiên cứu khoa học, công nghệ trọng điểm theo mô hình tiên tiến và triển khai những dự án khoa học, công nghệ quan trọng; nâng cao hiệu quả hoạt động các quỹ về phát triển khoa học, công nghệ, sử dụng hiệu quả nguồn vốn ngân sách nhà nước và có cơ chế, chính sách thu hút các nguồn lực ngoài nhà nước đầu tư nghiên cứu phát triển và ứng dụng khoa học, công nghệ. Có thể nói việc thu hút các nguồn lực khác nhau cho NC\&PT đã được Đảng và nhà nước coi là nhiệm vụ trọng tâm.

Đối với mỗi trường đại học, hai nhiệm vụ quan trọng nhất là truyền thụ kiến thức thông qua giảng dạy và sáng tạo tri thức thông qua NC\&PT. Hoạt động NC\&PT giúp các trường đại học phát triển các tư tưởng, hệ tiên đề, quy trình, công nghệ mới để thiết lập những nền móng căn bản của các ngành khoa học kỹ thuật của đất nước. Đào tạo và NC\&PT có mối quan hệ chặt chẽ với nhau. Đào tạo giúp truyền bá kiến thức từ NC\&PT trong khi đó thông qua NC\&PT, đào tạo tiếp cận đỉnh cao của tri thức, từ đó nâng lên chất lượng cao, trình độ cao hơn. Một cơ sở đào tạo đại học có uy tín vì vậy luôn phải xây dựng một nền tảng nghiên cứu và học thuật vững chắc. Trên thực tế, để NC\&PT cần hội tụ nhiều điều kiện như môi trường nghiên cứu, giảng dạy thuận lợi cho hoạt động NC\&PT; đảm bảo tự do học thuật; điều kiện về trang thiết bị nghiên cứu cùng các yếu tố về chính sách liên quan phù hợp. Đứng trước yêu cầu hội nhập, các cơ sở đại học ở Việt Nam đặc biệt là các trường đại học khối kỹ thuật, công nghệ cần phải nhận thức được các điều kiện cản trở, hạn chế hoạt động NC\&PT của mình, từ đó 
có các giải pháp phù hợp để thúc đẩy hoạt động này phát triển mạnh mẽ.

\section{Tổng quan nghiên cứu}

Trên thế giới nhiều học giả đã quan tâm tới việc tìm hiểu các nhân tố ảnh hưởng tới kết quả hoạt động nghiên cứu khoa học. Finkelstein (1984) đề xuất 7 nhân tố quan trọng ảnh hưởng tới tỷ lệ xuất bản của giảng viên đại học bao gồm định hướng nghiên cứu, bằng cấp cao nhất trong một lĩnh vực, các thói quen xuất bản từ khi trẻ tuổi, các hoạt động xuất bản trước đây, trao đổi thông tin với đồng nghiệp trong ngành, đăng ký và đọc nhiều tạp chí chuyên ngành và đủ thời gian dành cho nghiên cứu. Điểm hạn chế của nghiên cứu này là chưa đề cập tới các nhân tố của tổ chức trong việc ảnh hưởng tới năng suất nghiên cứu của giảng viên đại học.

Nghiên cứu của Creswell (1985) chia các nhân tố ảnh hưởng tới kết quả nghiên cứu của giảng viên thành 2 nhóm. Nhóm nhân tố cá nhân bao gồm cấp độ trong ngành, thời gian dành cho các hoạt động nghiên cứu, các hoạt động xuất bản khi còn trẻ, các phản hồi tích cực từ đồng nghiệp đối với các nỗ lực nghiên cứu, duy trì liên lạc thường xuyên với các đồng nghiệp đang nghiên cứu các chủ đề tương tự. Nhóm nhân tố tổ chức bao gồm quy mô và danh tiếng của trường đại học, sự trân trọng kết quả nghiên cứu của trường đại học, việc dành thời gian của trường đại học cho hoạt động nghiên cứu của giảng viên.

Dundar và Lewis (1998) sử dụng số liệu của Hội đồng nghiên cứu quốc gia Hoa Kỳ và chia các nhân tố ảnh hưởng thành nhóm nhân tố cá nhân và nhóm nhân tố môi trường. Nhóm nhân tố cá nhân bao gồm tính cách và kinh nghiệm cá nhân của giảng viên. Nhóm nhân tố môi trường liên quan đến tính chất sở hữu của trường đại học (đại học tư nhân hay đại học công), số lượng giáo sư, phần trăm giảng viên tích cực xuất bản trên các tạp chí, quy mô giảng viên.

Brocato (2001) sử dụng số liệu ở các trường đại học ở Hoa Kỳ phân chia các nhân tố này thành các hoạt động tương tác liên quan tới nghiên cứu khi còn trẻ, các tính chất về tâm lý và nhân khẩu học của các cá nhân, các nhân tố liên quan đến trường đại học và khoa chuyên môn nơi các giảng viên làm việc.

Bland và cộng sự (2002 và 2005) xem xét các nhân tố ảnh hưởng tới kết quả nghiên cứu của giảng viên tại các trường đại học Minnesota ở Hoa Kỳ và phân thành 2 nhóm chính: nhân tố cá nhân và tính chất quản lý của tổ chức. Cả 2 nhóm nhân tố này tạo nên tính chất đặc thù của trường đại học và từ đó ảnh hưởng tới năng suất nghiên cứu tổng thể của cả trường đại học.

Gần đây hơn, nghiên cứu của Dhillon và cộng sự (2015) nghiên cứu kết quả nghiên cứu của giảng viên tại trường đại học công nghệ Malaysia (UTM) và chia các nhân tố thành 3 nhóm: nhóm nhân tố cá nhân, nhóm nhân tố môi trường và nhóm nhân tố hành vi liên quan đến sự tương tác, trao đổi thông tin giữa các giảng viên đại học.

Một số nghiên cứu trước đây đã xem xét tác động của nhân tố tài chính tới việc thúc đẩy các hoạt động nghiên cứu và phát triển. Defazio và cộng sự (2009) đã xem xét việc tài trợ có điều kiện theo yêu cầu hợp tác ảnh hưởng đến hành vi hợp tác và năng suất của nhà nghiên cứu bằng cách sử dụng dữ liệu của 294 nhà nghiên cứu trong 39 mạng nghiên cứu của $\mathrm{EU}$ trong khoảng thời gian 15 năm. Các tác giả đã tìm thấy một tác động tích cực của tài trợ tài chính và hợp tác đến năng suất nghiên cứu. Bolli \& Somogyi (2011) đã phân tích tác động của các 
quỹ của bên thứ ba tư nhân và công cộng đến năng suất của các khoa đại học Thụy Sĩ và các tổ chức nghiên cứu công cộng. Các tác giả nhận thấy rằng cả tài trợ của bên thứ ba và tư nhân đều cải thiện năng suất xuất bản trong khi tài trợ tư nhân chủ yếu thúc đẩy năng suất chuyển giao công nghệ. Fedderke \& Goldschmidt (2014) đã so sánh kết quả nghiên cứu của các nhà nghiên cứu nhận được tài trợ so với những người có năng lực tương tự nhưng không nhận được tài trợ. Kết quả cho thấy tài trợ tài chính đã cải thiện đáng kể hiệu suất của nhà nghiên cứu. Muscio và cộng sự (2013) đã sử dụng dữ liệu tài chính cho toàn bộ các khoa của trường đại học Ý tham gia nghiên cứu về khoa học kỹ thuật và vật lý và tìm thấy bằng chứng rằng tài trợ tài chính của Chính phủ cho các trường đại học đóng vai trò bổ sung cho tài trợ từ các hợp đồng nghiên cứu và tư vấn và giúp thúc đẩy các trường đại học hợp tác với ngành công nghiệp. Nghiên cứu của Banal-Estanol và cộng sự (2015) cũng nhận thấy rằng sự sẵn có của các nguồn tài chính là chìa khóa thành công của các chương trình nghiên cứu ứng dụng.

Các nghiên cứu trước đây trên thế giới đã phân loại các nhân tố ảnh hưởng đến kết quả nghiên cứu của giảng viên nhưng hầu hết chỉ tập trung nghiên cứu tại các trường đại học của quốc gia phát triển, ít nghiên cứu sử dụng số liệu của nhiều trường đại học khác nhau tại một quốc gia đang phát triển như Việt Nam.

Các nghiên cứu ở Việt Nam tìm hiểu về các nhân tố ảnh hưởng tới kết quả nghiên cứu khoa học hoặc hoạt động phát triển khoa học và công nghệ còn rất ít. Các nghiên cứu chủ yếu tiếp cận ở góc độ vĩ mô như tìm hiểu một số giải pháp cơ bản nhằm phát triển thị trường khoa học - công nghệ ở Việt Nam (Phạm Văn Dũng, 2008), tìm hiểu về cơ chế tài chính cho hoạt động khoa học và công nghệ ở Việt Nam (Nguyễn Hồng Sơn, 2015), đưa ra các định hướng đẩy mạnh các hoạt động KH\&CN phục vụ sự nghiệp công nghiệp hóa hiện đại hóa đất nước (Phan Xuân Dũng, 2016), tìm hiểu vai trò của Nhà nước trong thúc đẩy hoạt động khoa học và công nghệ (Đặng Thu Giang, Cao Thu Anh, 2016), đưa ra các giải pháp nâng cao chất lượng hoạt động nghiên cứu khoa học trong các cơ sở giáo dục đại học (Phùng Văn Hiền, 2017) hoặc mô tả thực trạng đầu tư cho phát triển khoa học và công nghệ từ ngân sách Nhà nước (Nguyễn Hồ Phi Hà, 2018).

Các vấn đề về tài chính cho KH\&CN nói chung và NC\&PT nói riêng được đề cập trong một số nghiên cứu trước đây như tổng hợp khung pháp lý chung cho hoạt động của các quỹ, phân tích ưu nhược điểm của mỗi loại quỹ, chỉ ra các trở ngại đối với các tổ chức học thuật và doanh nghiệp trong việc khai thác các nguồn tài chính từ các quỹ đó, đưa ra gợi ý về cách thức mà các tổ chức học thuật và doanh nghiệp có thể áp dụng để phát triển các hoạt động nghiên cứu chung cũnghư những sửa đổi pháp lý cần thiết để tạo điều kiện cho hoạt động phối hợp giữa các tổ chức học thuật và doanh nghiệp trong việc khai thác nguồn quỹ (NguyễnĐăng Tuệ, 2016), giải pháp tài chính cho phát triển khoa học và công nghệ (Bùi Tiến Dũng2016) chỉ ra các loại quỹ KH\&CN hiện nay của Việt Nam, kinh nghiệm phát triển quỹ phát triển $\mathrm{KH} \& \mathrm{CN}$ của các trường đại học nước ngoài, từ đó đưa ra đề xuất khai thác quỹ phát triển $\mathrm{KH} \& \mathrm{CN}$ nhằm tăng cường sự tự chủ về hoạt động NC\&PT cho các trường đại học ở Việt Nam (NguyễnĐăng Tuệ \& Hứa Phương Linh, 2017). Kết quả của đề tài cấp Trường của Trường đại học Bách khoa Hà Nội (Nghiên cứu mô hình huy động và quản lý nguồn lực tài chính cho phát triển khoa 
học và công nghệ của Trường đại học Bách khoa Hà Nội mã số T2016 - PC -172) cũng cho thấy từ góc độ của các giảng viên, nhân tố tài chính được xác định là một nhân tố quan trọng ảnh hưởng đến hoạt động phát triển khoa học và công nghệ nói chung và hoạt động NC\&PT nói riêng. Tuy nhiên, trong số các nghiên cứu trước đây, chưa có nghiên cứu nào xem xét các vấn đề tài chính cho NC\&PT dưới góc nhìn của các cán bộ quản lý để trả lời câu hỏi những yếu tố nào đang thúc đẩy và cản trở hoạt động NC\&PT tại các trường đại học ở Việt Nam.

\section{Phương pháp và mô hình nghiên cứu}

Để đánh giá các nhân tố ảnh hưởng tới kết quả nghiên cứu khoa học và phát triển công nghệ của các trường đại học từ góc độ của cán bộ quản lý, tác giả sử dụng mô hình nghiên cứu dựa trên mô hình của Dhillon và cộng sự (2015). Theo đó, kết quả nghiên cứu khoa học của trường đại học chịu ảnh hưởng của các nhân tố liên quan đến năng lực của các cá nhân, tính chất của trường đại học và nhân tố tương tác của các giảng viên. Trên cơ sở mô hình này, tác giả đưa vào nhân tố nhân lực (tương đương với nhân tố cá nhân trong mô hình của Dhillon), nhân tố liên quan đến trao đổi thông tin (tương đương với nhân tố hành vi trong mô hình của Dhillon) và nhân tố quản lý (tương đương với nhân tố môi trường theo mô hình của Dhillon và cộng sự (2015)). Nhân tố tài chính được thêm vào mô hình do các phỏng vấn với các bên liên quan và các nghiên cứu liên quan ở Việt Nam trước đây cho thấy tài chính có thể là một rào cản đối với kết quả hoạt động khoa học và công nghệ của các trường đại học. Do vậy nhân tố này được xem xét dưới góc độ rào cản (tác động ngược chiều) đối với kết quả $\mathrm{KH} \& \mathrm{CN}$ của trường đại học trong khi các nhân tố khác được xem xét dưới góc độ nhân tố tác động cùng chiều. Mô hình nghiên cứu chi tiết được mô tả trên Hình 1.

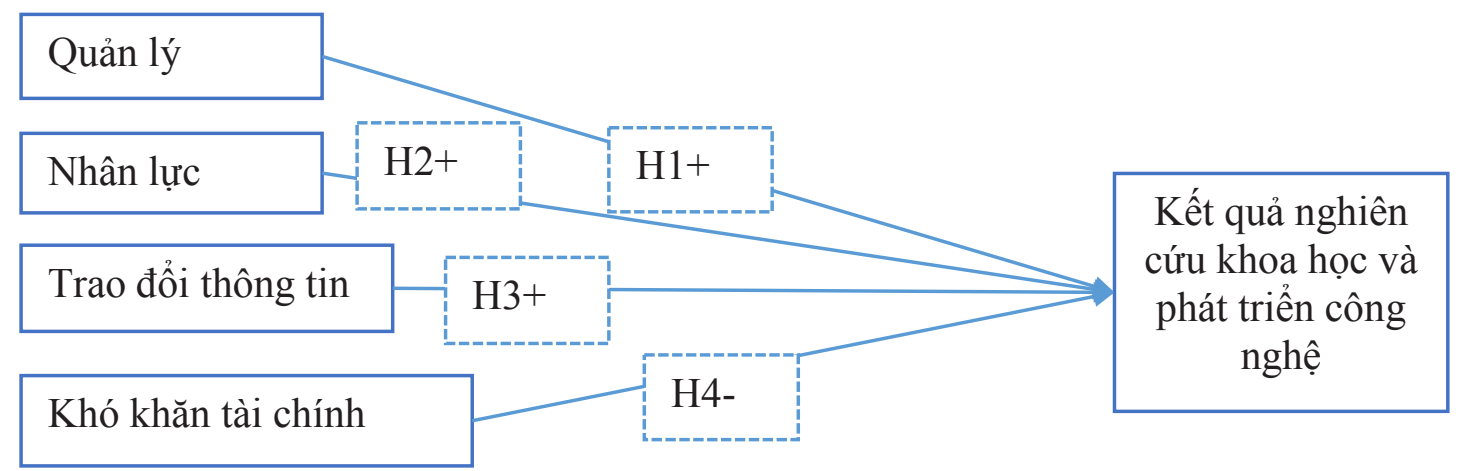

\section{Hình 1. Mô hình và các giả thuyết nghiên cứu}

Nguồn: Tác giả xây dụng dựa trên mô hình của Dhillon và cộng sụ (2015)

Mô hình nghiên cứu phản ánh sự đánh giá chủ quan từ phía các nhà quản lý của trường. Các giả thuyết nghiên cứu đối với mô hình này là các nhân tố (1) Quản lý (2) Trao đổi thông tin (3) Nhân lực tác động tích cực (cùng chiều) tới kết quả nghiên cứu khoa học chung của trường đại học $(\mathrm{H} 1 \sim \mathrm{H} 3)$. Nhân tố rào cản tài chính tạo ra các rào cản (tác động ngược chiều) tới kết quả nghiên cứu khoa học chung của trường đại học được đánh giá một cách chủ quan từ phía cán bộ quản lý của nhà trường (H4).

Các nhân tố được đo lường dựa trên kết quả của các bảng câu hỏi được thiết kế trên thang 
đo Likert 5 bậc theo đó 1 là Hoàn toàn đồng ý, 2 là Đồng ý, 3 là Trung lập, 4 là Không đồng ý, 5 là Hoàn toàn không đồng ý.

Thước đo chủ quan được xây dựng dựa trên đánh giá từ phía các cán bộ giảng viên và nhà quản lý của bản thân các trường đại học dựa trên 4 câu hỏi thể hiện sự đồng ý hay không đồng ý của người được hỏi với các nhận định (1) Kết quả NC\&PTKHCN đạt được mục tiêu do Trường đề ra (KQ1) (2) Kết quả NC\&PTKHCN phù hợp với năng lực của Trường? (KQ2) (3) Số lượng sản phẩm khoa học có chất lượng tốt được trường công bố trong 5 năm vừa qua có $x u$ hướng tăng lên (KQ3) (4) Các sản phẩm từ hoạt động NC\&PTKHCN của trường được ứng dụng tốt trong thực tế (KQ4). Thước đo này phản ánh sự đánh giá chủ quan từ phía những người thực hiện hoạt động NCKH\&PTCN và những người quản lý hoạt động này từ trường để đánh giá cụ thể xem những hoạt động NCKH\&PTCN đã đạt được mục tiêu, phù hợp với năng lực của trường, có số lượng ngày càng tăng và ứng dụng tốt cho thực tế hay không.

Nhóm nhân tố quản lý được đo lường bằng đánh giá từ phía các cán bộ giảng viên và nhà quản lý của bản thân các trường đại học dựa trên các câu hỏi thể hiện sự đồng ý hay không đồng ý của người được hỏi với các nhận định: Trường tạo điều kiện thuận lợi cho các hoạt động NC\&PTKHCN (QL1) Trường thường xuyên tổ chức các cuộc thi NC\&PTKHCN (QL2) Trường công bố công khai báo cáo định kì về số lượng công trình NC\&PTKHCN (QL3) Trường có chính sách đãi ngộ đặc biệt đối với nhà nghiên cứu có kết quả NC\&PTKHCN xuất sắc (QL4) Quy trình cấp vốn cho hoạt động NC\&PTKHCN của trường đơn giản (QL5) Trường có quy định rõ ràng liên quan đến việc đăng ký và tiếp nhận tài trợ ngoài Trường cho hoạt động NC\&PTKHCN của cán bộ giảng viên (QL6) Trường hỗ trợ cho cán bộ giảng viên trong việc hoàn thiện hồ sơ đăng ký xin tài trợ NC\&PTKHCN từ các nguồn ngoài Trường (QL7), Trường quản lý chặt chẽ cán bộ giảng viên trong việc đăng ký xin và nhận tài trợ ngoài Trường dành cho NC\&PTKHCN (QL8) Mức kinh phí đóng góp cho trường khi cán bộ giảng viên thực hiện hoạt động NC\&PTKHCN hợp tác với bên ngoài hợp lý (QL9), Quy trình cấp vốn cho hoạt động NC\&PTKHCN của trường minh bạch (QL10).

Nhóm nhân tố liên quan đến trao đổi thông tin được đo lường bằng đánh giá từ phía các cán bộ giảng viên và nhà quản lý của bản thân các trường đại học dựa trên các câu hỏithể hiện sự đồng ý hay không đồng ý của người được hỏvới các nhận định: Trường thiết lập tốt mối quan hệ với doanh nghiệp trong hoạt động NC\&PTKHCN (TT1), Giảng viên của trường thường xuyên trao đổi thông tin về học thuật (TT2), Trường cung cấp thông tin cho cán bộ giảng viên về các nguồn tài trợ ngoài Trường dành cho NC\&PTKHCN (TT3), Trường thường xuyên tổ chức các hội thảo về NC\&PTKHCN (TT4).

Nhóm nhân tố liên quan đến nhân lực được đo lường bằng đánh giá từ phía các cán bộ giảng viên và nhà quản lý của bản thân các trường đại học dựa trên các câu hỏi thể hiện sự đồng ý hay không đồng ý của người được hỏi với các nhận định: Số lượng giảng viên có năng lực nghiên cứu đủ đáp ứng nhu cầu NC\&PTKHCN của Trường (NL1), Giảng viên của trường được đào tạo về phương pháp NC\&PTKHCN (NL2), Giảng viên của trường được đào tạo về kỹ năng NC\&PTKHCN (NL3), Giảng viên của trường được giáo dục về đạo đức trong NC\&PTKHCN (NL4), Giảng viên của trường tuân thủ các 
nguyên tắc về đạo đức trong NC\&PTKHCN (NL5), Giảng viên của trường được đào tạo về cách thức tìm nguồn tài chính dành cho NC\&PTKHCN (NL6), Giảng viên của trường có uy tín trong việc thực hiện các hoạt động NC\&PTKHCN (NL7).

Nhóm nhân tố liên quan đến tài chính được đo lường bằng đánh giá từ phía các cán bộ giảng viên và nhà quản lý của bản thân các trường đại học dựa trên các câu hỏi thể hiện sự đồng ý hay không đồng ý của người được hỏiới các nhận định: Nhiều dự án NC\&PT KH\&CN của giảng viên không thực hiện được do không nhận được nguồn kinh phí hỗ trợ từ Trường (TC1), Nguồn kinh phí được cấp từ trường không đủ để thực hiện tốt các dự án NC\&PTKHCN (TC2), Trường thiếu cơ quan làm đầu mối để huy động nguồn lực tài chính từ các nguồn bên trong và ngoài trường dành cho hoạt động NC\&PTKHCN (TC3). Các thang đo của mô hình được trình bày chi tiết trong Bảng 1 .

Bảng 1. Thang đo của mô hình

\begin{tabular}{|c|c|c|c|}
\hline \begin{tabular}{c|} 
Thứ \\
tụ̣
\end{tabular} & Yếu tố & $\begin{array}{c}\text { Thang } \\
\text { đo }\end{array}$ & Câu hỏi \\
\hline 1. & \multirow{4}{*}{$\begin{array}{c}\text { Kết quả } \\
\text { nghiên cứu } \\
\text { khoa học } \\
\text { và phát } \\
\text { triển công } \\
\text { nghệ }\end{array}$} & KQ1 & Kết quả NC\&PTKHCN đạt được mục tiêu do Trường đề ra \\
\hline 2. & & KQ2 & Kết quả NC\&PTKHCN phù hợp với năng lực của Trường \\
\hline 3. & & KQ3 & $\begin{array}{l}\text { Số lượng sản phẩm khoa học có chất lượng tốt được trường công bố } \\
\text { trong } 5 \text { năm vừa qua có xu hướng tăng lên }\end{array}$ \\
\hline 4. & & KQ4 & $\begin{array}{l}\text { Các sản phẩm từ hoạt động NC\&PTKHCN của trường được ứng } \\
\text { dụng tốt trong thực tế }\end{array}$ \\
\hline 5. & \multirow{10}{*}{ Quản lý } & QL1 & Trường tạo điều kiện thuận lợi cho các hoạt động NC\&PTKHCN \\
\hline 6. & & QL2 & Trường thường xuyên tổ chức các cuộc thi NC\&PTKHCN \\
\hline 7. & & QL3 & $\begin{array}{l}\text { Trường công bố công khai báo cáo định kì về số lượng công trình } \\
\text { NC\&PTKHCN }\end{array}$ \\
\hline 8. & & QL4 & $\begin{array}{l}\text { Trường có chính sách đãi ngộ đặc biệt đối với nhà nghiên cứu có kết } \\
\text { quả NC\&PTKHCN xuất sắc }\end{array}$ \\
\hline 9. & & QL5 & $\begin{array}{l}\text { Quy trình cấp vốn cho hoạt động NC\&PTKHCN của trường đơn } \\
\text { giản }\end{array}$ \\
\hline 10. & & QL6 & $\begin{array}{l}\text { Trường có quy định rõ ràng liên quan đến việc đăng ký và tiếp nhận } \\
\text { tài trợ ngoài Trường cho hoạt động NC\&PTKHCN của cán bộ giảng } \\
\text { viên }\end{array}$ \\
\hline 11. & & QL7 & $\begin{array}{l}\text { Trường hỗ trợ cho cán bộ giảng viên trong việc hoàn thiện hồ sơ } \\
\text { đăng ký xin tài trợ NC\&PTKHCN từ các nguồn ngoài Trường }\end{array}$ \\
\hline 12. & & QL8 & $\begin{array}{l}\text { Trường quản lý chặt chẽ cán bộ giảng viên trong việc đăng ký xin và } \\
\text { nhận tài trợ ngoài Trường dành cho NC\&PTKHCN }\end{array}$ \\
\hline 13. & & QL9 & $\begin{array}{l}\text { Mức kinh phí đóng góp cho trường khi cán bộ giảng viên thực hiện } \\
\text { hoạt động NC\&PTKHCN hợp tác với bên ngoài hợp lý }\end{array}$ \\
\hline 14. & & QL10 & $\begin{array}{l}\text { Quy trình cấp vốn cho hoạt động NC\&PTKHCN của trường minh } \\
\text { bạch }\end{array}$ \\
\hline
\end{tabular}




\begin{tabular}{|c|c|c|c|}
\hline $\begin{array}{c}\text { Thứ } \\
\text { tụ̣ }\end{array}$ & Yếu tố & $\begin{array}{c}\text { Thang } \\
\text { do }\end{array}$ & Câu hỏi \\
\hline 15. & \multirow{4}{*}{$\begin{array}{l}\text { Trao đổi } \\
\text { thông tin }\end{array}$} & TT1 & $\begin{array}{l}\text { Trường thiết lập tốt mối quan hệ với doanh nghiệp trong hoạt động } \\
\text { NC\&PTKHCN }\end{array}$ \\
\hline 16. & & TT2 & Giảng viên của trường thường xuyên trao đổi thông tin về học thuật \\
\hline 17. & & TT3 & $\begin{array}{l}\text { Trường cung cấp thông tin cho cán bộ giảng viên về các nguồn tài } \\
\text { trợ ngoài Trường dành cho NC\&PTKHCN }\end{array}$ \\
\hline 18. & & TT4 & Trường thường xuyên tổ chức các hội thảo về NC\&PTKHCN \\
\hline 19. & \multirow{7}{*}{ Nhân lực } & NL1 & $\begin{array}{l}\text { Số lượng giảng viên có năng lực nghiên cứu đủ đáp ứng nhu cầu } \\
\text { NC\&PTKHCN của Trường }\end{array}$ \\
\hline 20. & & NL2 & Giảng viên của trường được đào tạo về phương pháp NC\&PTKHCN \\
\hline 21. & & NL3 & Giảng viên của trường được đào tạo về kỹ năng NC\&PTKHCN \\
\hline 22. & & NL4 & Giảng viên của trường được giáo dục về đạo đức trong NC\&PTKHCN \\
\hline 23. & & NL5 & $\begin{array}{l}\text { Giảng viên của trường tuân thủ các nguyên tắc về đạo đức trong } \\
\text { NC\&PTKHCN }\end{array}$ \\
\hline 24. & & NL6 & $\begin{array}{l}\text { Giảng viên của trường được đào tạo về cách thức tìm nguồn tài chính } \\
\text { dành cho NC\&PTKHCN }\end{array}$ \\
\hline 25. & & NL7 & $\begin{array}{l}\text { Giảng viên của trường có uy tín trong việc thực hiện các hoạt động } \\
\text { NC\&PTKHCN }\end{array}$ \\
\hline 26. & \multirow{3}{*}{ Tài chính } & TC1 & $\begin{array}{l}\text { Nhiều dự án NC\&PT KH\&CN của giảng viên không thực hiện được } \\
\text { do không nhận được nguồn kinh phí hỗ trợ từ Trường }\end{array}$ \\
\hline 27. & & TC2 & $\begin{array}{l}\text { Nguồn kinh phí được cấp từ trường không đủ để thực hiện tốt các dự } \\
\text { án NC\&PTKHCN }\end{array}$ \\
\hline 28 & & TC3 & $\begin{array}{l}\text { Trường thiếu cơ quan làm đầu mối đề huy động nguồn lực tài } \\
\text { chính từ các nguồn bên trong và ngoài trường dành cho hoạt động } \\
\text { NC\&PTKHCN }\end{array}$ \\
\hline
\end{tabular}

Nguồn: Tác giả tổng hơp

Bài nghiên cứu được thực hiện sử dụng mô hình cấu trúc tuyến tính (SEM) và áp dụng các phương pháp phân tích nhân tố khám khá (EFA) và phân tích nhân tố khẳng định $(\mathrm{CFA})$ trên phần mềm Stata 14.

\section{Số liệu nghiên cứu}

Từ ngày 16/5 đến 25/6/2018, hoạt động khảo sát được tiến hành trên 115 đại học và trường đại học khối ngành kỹ thuật và công nghệ ở Việt Nam kết hợp với Ban khoa học và Dịch vụ, Hiệp hội các trường Đại học và Cao đẳng Việt Nam. Hình thức khảo sát được thực hiện qua 2 kênh: khảo sát trực tiếp và gửi bảng hỏi. Các trường đại học được chọn để khảo sát là những trường đại học có đào tạo các ngành liên quan đến kỹ thuật và công nghệ. Các nhà quản lý được lấy ý kiến trong cuộc khảo sát là những cán bộ quản lý cấp cao của trường như hiệu trưởng, phó hiệu trưởng, trưởng các phòng ban có liên quan đến hoạt động nghiên cứu và phát triển. Mặc dù vậy, các ý kiến được thu thập thể hiện quan điểm cá nhân của những người được khảo sát và điều này được nêu rõ trong bảng hỏi. Tổng số bảng khảo sát được phát ra đối với cán bộ quản lý là 252, thu về 
227, số bảng đầy đủ thông tin và sử dụng được cho nghiên cứu là 217 , chiếm tỷ lệ $86.1 \%$.

Mục đích chính của bảng khảo sát là xác định sự cần thiết của quỹ phát triển khoa học và công nghệ của trường và các yếu tố ảnh hưởng đến kết quả khoa học công nghệ của trường đại học. Tác giả sử dụng những biến liên quan trong bộ dữ liệu thu thập được để thực hiện nghiên cứu này.

\section{Kết quả nghiên cứu}

\subsection{Kiểm định độ tin cậy}

a. Nguyên tắc kiểm định độ tin cậy của thang đo và kiểm định khám phá

Độ tin cậy của thang đo được đánh giá bằng phương pháp nhất quán nội tại qua hệ số Cronbach's Alpha trước khi phân tích nhân tố EFA để loại các biến không phù hợp (Nguyễn Đình Thọ \& Nguyễn Thị Mai Trang, 2009). Hệ số tin cậy Cronbach's Alpha chỉ cho biết các đo lường có liên kết với nhau hay không; nhưng không cho biết biến quan sát nào cần bỏ đi và biến quan sát nào cần giữ lại. Khi đó, việc tính toán hệ số tương quan giữa biến-tổng sẽ giúp loại ra những biến quan sát nào không đóng góp nhiều cho sự mô tả của khái niệm cần đo (Hoàng Trọng \& Chu Nguyễn Mộng Ngọc, 2005). Các tiêu chí được sử dụng khi thực hiện đánh giá độ tin cậy thang đo bao gồm:

Loại các biến quan sát có hệ số tương quan biến-tổng nhỏ (nhỏ hơn 0,6): Các mức giá trị của Alpha lớn hơn 0,8 là thang đo lường tốt; từ 0,7 đến 0,8 là sử dụng được; từ 0,6 trở lên là có thể sử dụng trong trường hợp khái niệm nghiên cứu là mới hoặc là mới trong bối cảnh nghiên cứu (Hoàng Trọng \& Chu Nguyễn Mộng Ngọc, 2005).

\section{b. Kiểm định thang đo}

Số quan sát trong mô hình là 217. Thang đo của mô hình được điều chỉnh thông qua việc kiểm tra giá trị của độ tin cậy Alpha kết hợp với ma trận xoay nhân tố.

Các nhân tố quản lý được điều chỉnh và loại biến theo nguyên tắc đề cập ở trên được trình bày trong Bảng 2.

Bảng 2. Độ tin cậy của nhóm nhân tố quản lý trước và sau khi điều chỉnh

\begin{tabular}{|l|c|c|}
\hline \multicolumn{1}{|c|}{ Nhân tố } & Trước khi điều chỉnh & Sau khi điều chỉnh \\
\hline QL1 & Alpha nếu loại bỏ nhân tố & Alpha nếu loại bỏ nhân tố \\
\hline QL2 & 0.6918 & 0.8092 \\
\hline QL3 & 0.6866 & 0.8146 \\
\hline QL4 & 0.6942 & 0.8210 \\
\hline QL5 & 0.6860 & 0.8116 \\
\hline QL6 & 0.6982 & 0.8253 \\
\hline QL7 & 0.7564 & Đã loại bỏ khi điều chỉnh \\
\hline QL8 & 0.6937 & 0.8143 \\
\hline QL9 & 0.7598 & Đã loại bỏ khi điều chỉnh \\
\hline QL10 & 0.7828 & Đã loại bỏ khi điều chỉnh \\
\hline Cronbach Alpha của nhóm nhân tố & 0.7095 & 0.832 \\
\hline
\end{tabular}


Sau khi kiểm định và loại bỏ các nhân tố không phù hợp (QL6, QL8, QL9) thang đo có độ tin cậy alpha 0.8401 phù hợp để thực hiện mô hình.

Việc điều chỉnh các nhân tố trao đổi thông tin được trình bày trong Bảng 3 .

Bảng 3. Độ tin cậy của nhóm nhân tố trao đổi thông tin trước và sau khi điều chỉnh

\begin{tabular}{|l|c|c|}
\hline & $\begin{array}{c}\text { Trước khi } \\
\text { điều chỉnh }\end{array}$ & $\begin{array}{c}\text { Sau khi } \\
\text { điều chỉnh }\end{array}$ \\
\hline Nhân tố & $\begin{array}{c}\text { Alpha nếu loại } \\
\text { bỏ nhân tố }\end{array}$ & $\begin{array}{c}\text { Alpha nếu loại } \\
\text { bỏ nhân tố }\end{array}$ \\
\hline TT1 & 0.7698 & 0.8109 \\
\hline TT2 & 0.8791 & 0.8641 \\
\hline TT3 & 0.7674 & 0.7977 \\
\hline TT4 & 0.8012 & 0.8168 \\
\hline QL6 & & 0.8549 \\
\hline $\begin{array}{l}\text { Cronbach } \\
\text { Alpha } \\
\text { của nhóm } \\
\text { nhân tố }\end{array}$ & $\mathbf{0 . 8 5 4 9}$ & $\mathbf{0 . 8 6 2 5}$ \\
\hline
\end{tabular}

Nguồn: Tác giả tính toán

Các nhân tố thông tin gốc có Cronbach alpha khá cao. Kết hợp với kết quả ma trận xoay nhân tố (Bảng 6), nhân tố QL6 có nội dung liên quan đến vấn đề trao đổi thông tin NC\&PT nên được tích hợp với nhóm các nhân tố trao đổi thông tin. Kết quả có Cronbach alpha cao hơn cho thang đo và được sử dụng.

Các nhân tố nhân lực được điều chỉnh như được trình bày trong Bảng 4 .
Bảng 4. Độ tin cậy của nhóm nhân tố nhân lực trước và sau khi điều chỉnh

\begin{tabular}{|c|c|c|}
\hline & $\begin{array}{l}\text { Trước khi } \\
\text { điều chỉnh }\end{array}$ & $\begin{array}{c}\text { Sau khi } \\
\text { điều chỉnh }\end{array}$ \\
\hline Nhân tố & $\begin{array}{c}\text { Alpha nếu loại } \\
\text { bỏ nhân tố }\end{array}$ & $\begin{array}{c}\text { Alpha nếu loại } \\
\text { bỏ nhân tố }\end{array}$ \\
\hline NL1 & 0.6334 & 0.7175 \\
\hline NL2 & 0.6796 & $\begin{array}{l}\text { Đã loại bỏ khi } \\
\text { điều chỉnh }\end{array}$ \\
\hline NL3 & 0.6739 & 0.7764 \\
\hline NL4 & 0.7888 & $\begin{array}{l}\text { Đã loại bỏ khi } \\
\text { điều chỉnh }\end{array}$ \\
\hline NL5 & 0.6874 & 0.8056 \\
\hline NL6 & 0.76 & $\begin{array}{l}\text { Đã loại bỏ khi } \\
\text { điều chỉnh }\end{array}$ \\
\hline NL7 & 0.664 & 0.7394 \\
\hline $\begin{array}{l}\text { Cronbach } \\
\text { Alpha } \\
\text { của nhóm } \\
\text { nhân tố }\end{array}$ & 0.7355 & 0.8115 \\
\hline
\end{tabular}

Các nhân tố nhân lực có Cronbach Alpha 0.7355. Sau khi điều chỉnh loại bỏ một số nhân tố và dựa vào kết quả ma trận xoay (Bảng 7), kết quả của nhóm nhân tố được sử dụng có giá trị alpha 0.8115 .

Việc điều chỉnh các nhân tố tài chính được trình bày trên Bảng 5 . Dựa vào kết quả ma trận xoay nhân tố, nhân tố NL6 (liên quan đến vấn đề tài chính) được nhóm cùng với nhóm các nhân tố tài chính với thang đo có Cronbach alpha 0.7763 lớn hơn alpha của nhóm nhân tố ban đầu (0.7632). 
Bảng 5. Độ tin cậy của nhóm nhân tố tài chính trước và sau khi điều chỉnh

\begin{tabular}{|l|r|r|}
\hline & \multicolumn{1}{|c|}{$\begin{array}{c}\text { Trước khi } \\
\text { điều chỉnh }\end{array}$} & \multicolumn{1}{c|}{$\begin{array}{c}\text { Sau khi } \\
\text { điều chỉnh }\end{array}$} \\
\hline Nhân tố & $\begin{array}{c}\text { Alpha nếu loại } \\
\text { bỏ nhân tố }\end{array}$ & $\begin{array}{c}\text { Alpha nếu loại } \\
\text { bỏ nhân tố }\end{array}$ \\
\hline TC1 & 0.6119 & 0.656 \\
\hline TC2 & 0.7061 & 0.7191 \\
\hline TC3 & 0.7227 & 0.7402 \\
\hline NL6 & & 0.7632 \\
\hline $\begin{array}{l}\text { Cronbach } \\
\text { Alpha } \\
\text { của nhóm } \\
\text { nhân tố }\end{array}$ & $\mathbf{0 . 7 6 3 2}$ & $\mathbf{0 . 7 7 6 3}$ \\
\hline
\end{tabular}

Nguồn: Tác giả tính toán

Cuối cùng, độ tin cậy của nhóm nhân tố kết quả nghiên cứu và phát triển được trình bày trên
Bảng 6. Kết quả cho thấy giá trị Alpha tổng là phù hợp và có thể sử dụng cho mô hình.

Bảng 6. Độ tin cậy của nhóm nhân tố kết quả nghiên cứu và phát triển

\begin{tabular}{|l|r|}
\hline Nhân tố & Alpha của nhân tố \\
\hline KQ1 & 0.6885 \\
\hline KQ2 & 0.7563 \\
\hline KQ3 & 0.7645 \\
\hline KQ4 & 0.7304 \\
\hline $\begin{array}{l}\text { Cronbach Alpha của } \\
\text { nhóm nhân tố }\end{array}$ & 0.7884 \\
\hline
\end{tabular}

Nguồn: Tác giả tính toán

Ma trận xoay các nhân tố được trình bày trong Bảng 6 theo đó các ô trống thể hiện các nhân tố có giá trị tuyệt đối của hệ số tải nhỏ hơn 0.5. Giá trị của thước đo Kaiser-Meyer-Olkin của sự phù hơp chung của mẫu là 0.8063 lớn hơn 0.8 cho thấy việc lấy mẫu là phù hợp.

Bảng 7. Ma trận xoay các nhân tố

\begin{tabular}{|c|c|c|c|c|}
\hline Nhân tố & Nhóm nhân tố 1 & Nhóm nhân tố 2 & Nhóm nhân tố 3 & Nhóm nhân tố 4 \\
\hline QL1 & & 0.7973 & & \\
\hline QL2 & & 0.6648 & & \\
\hline QL3 & & 0.6268 & & \\
\hline QL4 & & 0.6903 & & \\
\hline QL5 & & 0.5967 & & \\
\hline QL6 & 0.7272 & & & \\
\hline QL7 & & 0.701 & & \\
\hline \multicolumn{5}{|l|}{ QL8 } \\
\hline \multicolumn{5}{|l|}{ QL9 } \\
\hline QL10 & & 0.5402 & & \\
\hline TT1 & 0.9087 & & & \\
\hline TT2 & 0.6966 & & & \\
\hline TT3 & 0.8331 & & & \\
\hline TT4 & 0.7723 & & & \\
\hline NL1 & & & 0.8054 & \\
\hline NL2 & & & 0.6795 & \\
\hline
\end{tabular}




\begin{tabular}{|l|r|r|r|r|}
\hline \multicolumn{1}{|c|}{ Nhân tố } & Nhóm nhân tố 1 & Nhóm nhân tố 2 & Nhóm nhân tố 3 & Nhóm nhân tố 4 \\
\hline NL3 & & & 0.6544 & \\
\hline NL4 & & & & \\
\hline NL5 & & & 0.6583 & \\
\hline NL6 & & & & 0.5699 \\
\hline NL7 & & & & 0.7299 \\
\hline TC1 & & & & 0.6694 \\
\hline TC2 & & & & \\
\hline TC3 & & & & 0.6395 \\
\hline
\end{tabular}

Nguồn: Tác giả tính toán

\subsection{Kiểm định các giả thuyết nghiên cứu}

a. Kết quả mô hình

Bảng 8. Kết quả hồi quy theo nhóm các nhân tố

\begin{tabular}{|l|l|r|r|r|r|}
\hline Giả thuyết & \multicolumn{1}{|c|}{ Nhân tố } & Giá trị hệ số Beta & \multicolumn{1}{c|}{$\begin{array}{c}\text { Phương sai } \\
\text { tiêu chuẩn }\end{array}$} & Hệ số z & Giá trị p \\
\hline H1+ & Quản lý & 0.20042 & 0.073613 & 2.72 & 0.006 \\
\hline H2+ & Nhân lực & 0.130433 & 0.077018 & 1.69 & 0.09 \\
\hline H3+ & Trao đổi thông tin & 0.055578 & 0.072383 & 0.77 & 0.443 \\
\hline H4- & Tài chính & -0.42289 & 0.069869 & -6.05 & 0 \\
\hline
\end{tabular}

Nguồn: Tác giả tính toán

Kết quả mô hình cho thấy các nhân tố khác đều có dấu tương ứng với chiều tác động của mô hình. Về mặt ý nghĩa thống kê, các nhân tố quản lý và tài chính có giá trị $\mathrm{p}$ nhỏ hơn 0.001 và có ý nghĩa thống kê ở mức 99\%, nhân tố nhân lực có giá trị p nhỏ hơn 0.1 , nhân tố trao đổi thông tin có giá trị $p$ khá lớn. Như vậy có bằng chứng để chấp nhận giả thuyết nghiên cứu số 1,2 và 4 , chưa có bằng chứng để chấp nhận giả thuyết nghiên cứu số 3 .

\section{b. Độ phù hợp của mô hình}

Các chỉ số của mô hình đều có kết quả khá tốt. Chỉ số RMSEA (Root mean squared error of approximation) có kết quả là 0.046 nhỏ hơn ngưỡng cho phép 0.05 . Giá trị $p$ thể hiện xác suất RMSEA $<=0.05$ là 0.791 lớn hơn ngưỡng cho phép 0.05. Các chỉ số CFI (Comparative fit index) là 0.926, TLI (Tucker-Lewis index là 0.92 đều cao hơn ngưỡng cho phép 0.9. Do vậy kết quả của mô hình có thể tin cậy và sử dụng được. Chỉ số AIC (Akaike’s information criterion) là 16830.259 và $\mathrm{BIC}$ (Bayesian information criterion) là 17137.829 đều khá thấp cho thấy mô hình được sử dụng tốt hơn các mô hình tương đương.

\section{Kết luận và hàm ý chính sách}

Kết quả nghiên cứu cho thấy các nhóm nhân tố quản lý, nhân lực có ảnh hưởng tới kết quả hoạt động phát triển khoa học và công nghệ và 
nhóm nhân tố tài chính là cản trở đối với hoạt động này. Trên cơ sở này tác giả đề xuất một số hàm ý chính sách dưới đây

Mô hình phản ánh ý kiến của các nhà quản lý cho thấy công tác quản lý đối với hoạt động NC\&PT nếu thực hiện tốt có thể đem lại những kết quả tích cực. Luật Giáo dục Đại học hiện hành đã quy định cơ sở giảng dạy cũng chính là cơ sở nghiên cứu khoa học. Chính vì vậy, để tăng cường hoạt động NC\&PT của các trường, bản thân các trường cần có phương thức quản lý hiệu quả, tạo điều kiện thuận lợi cho các hoạt động này thông qua việc tổ chức các cuộc thi, đưa ra các đãi ngộ đặc biệt với nhà nghiên cứu có kết quả xuất sắc. Trường cần có quy trình, quy định rõ ràng, minh bạch liên quan đến các hoạt động NC\&PT, công bố công khai báo cáo định kì về số lượng công trình NC\&PTKHCN. Trường hỗ trợ cho cán bộ giảng viên trong việc hoàn thiện hồ sơ đăng ký xin tài trợ NC\&PTKHCN từ các nguồn ngoài Trường.

Năng lực của đội ngũ giảng viên cũng là yếu tố quan trọng ảnh hưởng đến kết quả NC\&PT của các trường đại học. Kết quả này cũng tương thích với các kết quả nghiên cứu trước đây trên thế giới như Bland và cộng sự (2005) và Dhillon và cộng sự (2015). Việc nâng cao chất lượng đội ngũ nghiên cứu có thể được thực hiện thông qua việc tạo điều kiện cho giảng viên học tập, nâng cao trình độ, tạo môi trường tự do học thuật để các nhà khoa học có thể phát huy được tối đa năng lực của bản thân. Đặc biệt, một trong những biện pháp có thể áp dụng để tăng chất lượng đội ngũ nhà nghiên cứu, giảng viên trong các trường đại học là thành lập các nhóm nghiên cứu. Nhóm nghiên cứu giúp tập hợp các nhà khoa học có chuyên môn gần để phát triển môi trường học thuật chuyên sâu để phát triển ý tưởng khoa học, xây dựng trường phái học thuật, hoặc thu hút các nhà khoa học của nhiều ngành khác nhau để tập trung trí tuệ và sức lực giải quyết một vấn đề khoa học liên ngành. Đồng thời, hoạt động đào tạo và phát triển đội ngũ NC\&PT cũng được thông qua hoạt động của chính các nhóm nghiên cứu. Nhóm nghiên cứu có vai trò cực kỳ quan trọng trong việc triển khai từ nghiên cứu đến thử nghiệm, thúc đẩy sự ra đời của các phát minh, sáng chế và sản phẩm mới trong trường đại học. Vì vậy, một trong những giải pháp để nâng cao hiệu quả của các hoạt động nghiên cứu là phải xây dựng và phát triển được các nhóm nghiên cứu mạnh, đào tạo được đội ngũ cán bộ khoa học có trình độ và năng lực nghiên cứu khoa học tốt, có tâm huyết và kinh nghiệm trong nghiên cứu và đào tạo.

Cùng với việc thúc đẩy môi trường khoa học - đào tạo chung, các trường cần có chính sách phát triển một số lĩnh vực NC\&PT riêng được coi là thế mạnh. Các trường đại học cần có kế hoạch hình thành một đội ngũ các nhà khoa học đầu ngành cho từng lĩnh vực NC\&PT và đặt trọng tâm của nguồn nhân lực NC\&PT là đội ngũ nghiên cứu này. Trong các lĩnh vực có điều kiện tốt về cơ sở vật chất, trang thiết bị, phòng thí nghiệm tiên tiến, các trường đại học khối kỹ thuật và công nghệ có thể thu hút thêm nhà khoa học người Việt Nam hoặc người nước ngoài có uy tín để giao nhiệm vụ tập trung xây dựng lực lượng khoa học đủ mạnh hướng tới triển khai toàn diện nghiên cứu và đào tạo, chuyển giao công nghệ chất lượng quốc tế.

Từ kết quả nghiên cứu có thể thấy vấn đề tài chính đang là một nút thắt cản trở hoạt động NC\&PT. Để xây dựng năng lực NC\&PT của các trường đại học khối kỹ thuật và công nghệ, các trường đại học này cần phải thiết lập nền tảng vật chất hiện đại cùng năng lực tài chính tự chủ, vững mạnh. Một trong những giải pháp 
có thể thực hiện để tăng cường nguồn tài chính cho hoạt động NC\&PT của các trường đại học là thành lập quỹ phát triển $\mathrm{KH \& CN}$. Các quỹ phát triển KH\&CN ở các đại học ở Việt Nam đã được thành lập tại Đại học Quốc gia Hà Nội, Đại học Quốc gia Thành phố Hồ Chí Minh và trường Đại học Tôn Đức Thắng. Các quỹ này đã huy động được nguồn lực, duy trì và phát triển tốt các hoạt động của mình, tài trợ được nhiều dự án nghiên cứu khoa học và phát triển công nghệ, góp phần đưa 3 đại học có quỹ trở thành các đại học có số lượng công bố quốc tế hàng đầu Việt Nam (Nguyễn Đăng Tuệ, 2019). Đây có thể là một giải pháp đột phá giúp cho các trường đại học khối kỹ thuật và công nghệ thoát khỏi nút thắt về tài chính.

Bên cạnh giải pháp nói trên, các trường đại học cần đồng thời tạo các cơ chế đột phá, thay đổi, điều chỉnh cách đầu tư và hợp tác; thay đổi mô hình đầu tư cho NC\&PT của các trường đại học hướng tới sản phẩm đầu ra của NC\&PT; thay đổi phương thức tổ chức nhiệm vụ KH\&CN (các đề tài, dự án cấp bộ) trong các trường đại học theo dạng chương trình nghiên cứu gắn với mục tiêu phát triển của quốc gia, tránh đầu tư dàn trải. Việc đầu tư cho các chương trình nghiên cứu phải hướng tới tạo ra sản phẩm phục vụ đào tạo nhân lực và ứng dụng trực tiếp cho xã hội, gắn với xu thế KH\&CN của thế giới hiện nay.

\section{Hạn chế của nghiên cứu}

Nghiên cứu này còn có một số hạn chế sau đây. Thứ nhất, nghiên cứu chỉ phản ánh được kết quả đánh giá từ góc nhìn từ phía nhà quản lý NC\&PT tại các trường đại học. Đánh giá này có thể có sự khác biệt so với góc nhìn của giảng viên và những nhà tạo lập chính sách. Thứ hai, nghiên cứu này dựa trên đánh giá chủ quan của cán bộ quản lý. Kết quả cần có sự so sánh với các thước đo khách quan khác đối với hoạt động NC\&PT của trường đại học như số lượng công bố khoa học của các trường, tổng chi tiêu cho NC\&PT của các trường. Tuy nhiên hiện ở Việt Nam chưa có các số liệu chính xác đối với các chỉ tiêu này. Cuối cùng, nghiên cứu mới chỉ thực hiện ở Việt Nam và số liệu lấy cho riêng năm 2018. Cần có nghiên cứu so sánh sự thay đổi qua từng năm để làm rõ xu thế cũng như mở rộng phạm vi nghiên cứu để so sánh với các quốc gia khác.

\section{Tài liệu tham khảo}

\section{Tiếng Anh}

Banal-Estanol, A., Jofre-Bonet, M., \& Lawson, C. (2015). The double-edged sword of industry collaboration: Evidence from engineering academics in the UK. Research Policy, 44, 11601175.

Bland, C. J. và những tác giả khác, 2005. A Theoretical, Practical, Predictive Model of Faculty and Department Research Productivity. Academic Medicine, 80(3), pp. 225-237.

Bland, C. J., Seaquist, E., J. T. Pacala, B. C. \& Finstad, D., 2002. One school's strategy to assess and improve the vitality of its faculty. Academic Medicine, Số 77, pp. 368-376.

Bolli, T., Olivares, M., Bonaccorsi, A., Daraio, C., Aracil, A. G., \& Lepori, B. (2016). The differential effects of competitive funding on the production frontier and the efficiency of universities. Economics of Education Review, 1-14. doi:http://dx.doi.org/10.1016/j.econedurev.2016.01.007 
Brocato, J. J., 2001. The research productivity of family medicine department faculty: a national study dissertation., Michigan: Michigan State University.

Creswell, J. W., 1985. Faculty Research Performance: Lessons from the Sciences and Social Sciences, Washington, DC: Association for the Study of Higher Education.

Defazio, D., Lockett, A., \& Wright, M. (2009). Funding incentives, collaborative dynamics and scientific productivity: Evidence from the EU framework program. Research Policy, 38, 293305. doi:10.1016/j.respol.2008.11.008

Dhillon, S. K., Ibrahim, R. \& Selamat, A., 2015. Factors associated with scholarly publication productivity among academic staff: Case of a Malaysian public university. Technology in Society, Số 42, pp. 160-166.

Dundar, H. \& Lewis, D. R., 1998. Determinants of research productivity in higher education. Research Higher Education, Số 39, p. 607-31.

Fedderke, J., \& Goldschmidt, M. (2014). Does massive funding support of researchers work?: Evaluating the impact of the South African research chair funding initiative. Research Policy, 44(2), 467-482.

Finkelstein, M. J., 1984. The American Academic Profession: A Synthesis of Social Scientific Inquiry since World War II. Columbus: Ohio State University Press.

Muscio, A., Quaglione, D., \& Vallanti, G. (2013). Does government funding complement or substitute private research funding to universities? Research Policy, 42, 63-75.

\section{Tiếng Việt}

Bùi Tiến Dũng, 2016. Thêm nhiều giải pháp tài chính cho phát triển khoa học và công nghệ. Tạp chí Tài chính, Số 1-2016.

Đặng Thu Giang \& Cao Thu Anh, 2016. Vai trò của Nhà nước trong thúc đẩy hoạt động khoa học và công nghệ. Tạp chí Tài chính, 1(3), pp. 64-66.

Hoàng Trọng \& Chu Nguyễn Mộng Ngọc, 2005. Phân Tích Dữ Liệu Nghiên Cứu Với Spss. Thành phố Hồ Chí Minh: Nhà xuất bản Hồng Đức.

Nguyễn Đăng Tuệ, 2016. Legal issues concerning academic-industry collaboration in exploiting research funds. ICECH2016 - International Conference on Emerging Challenges: Partnership Enhancement, 2 11, pp. 91-99.

Nguyễn Đăng Tuệ, 2019. Áp dụng mô hình quỹ phát triển khoa học và công nghệ tại các trường đại học khối công nghệ ở Việt Nam. Tạp chí Khoa học và Công nghệ Trường đại học Công nghiệp, Số 50 .

Nguyễn Đăng Tuệ \& Hứa Phương Linh, 2017. Mô hình quỹ khoa học công nghệ với sự tự chủ tài chính trong hoạt động nghiên cứu của các trường đại học công nghệ. Hội thảo Quốc gia Tự chủ đại học - Cơ hội và thách thức, 7 3, pp. 112-127.

Nguyễn Đình Thọ \& Nguyễn Thị Mai Trang , 2009. Nghiên Cứu Khoa Học Trong Quản Trị Kinh Doanh. Hà Nội: Nhà xuất bản Thống kê.

Nguyễn Hồ Phi Hà, 2018. Tạp chí Tài chính. [Trực tuyến] tại địa chỉ: http://tapchitaichinh.vn/ nghien-cuu--trao-doi/trao-doi-binh-luan/thuc-trang-dau-tu-cho-phat-trien-khoa-hoc-va-congnghe-tu-ngan-sach-nha-nuoc-133809.html [Đã truy cập 11 2018]. 
Nguyễn Hồng Sơn, 2012. Cơ chế tài chính cho hoạt động khoa học và công nghệ ở Việt Nam: Một số hạn chế và giải pháp hoàn thiện. Những vấn đề kinh tế và chính trị thế giới, 6(194), pp. $57-$ 66.

Phạm Văn Dũng, 2008. Một số giải pháp cơ bản nhằm phát triển thị trường khoa học - công nghệ ở Việt Nam. Tạp chí Khoa học ĐHQGHN, Kinh tế - Luật, Số 24, pp. 35-48.

Phan Xuân Dũng, 2016. Đẩy mạnh hoạt động khoa học và công nghệ phục vụ sự nghiệp công nghiệp hóa, hiện đại hóa đất nước. Tạp chí Cộng sản.

Phùng Văn Hiền, 2017. Giải pháp nâng cao chất lượng hoạt động nghiên cứu khoa học trong các cơ sở giáo dục đại học. Tạp chí Lý luận Chính trị, Số 4-2017. 